\title{
Stock Prices, News, and Economic Fluctuations: Comment*
}

\author{
André Kurmann \\ Elmar Mertens \\ Federal Reserve Board Federal Reserve Board \\ Online Appendix
}

November 7, 2013

\begin{abstract}
This web appendix provides some more analytical details as well as additional results to our comment.
\end{abstract}

*The views expressed in this paper do not necessarily represent the views of the Federal Reserve System or the Federal Open Market Committee. 


\section{Contents}

\begin{tabular}{|l|l|}
\hline A The VECM's VMA representation & 3
\end{tabular}

A.1 C(1) has rank 1 . . . . . . . . . . . . . . . . 4

A.2 Long-run shocks to TFP in the VECM . . . . . . . . . 6

B Multiple BP shock candidates 8

B.] The entire set of solutions to the BP scheme . . . . . . . . 9

B.2 Additional results from the BP VECMS . . . . . . . . . . . 11 


\section{A The VECM's VMA representation}

This section derives the vector moving average (VMA) representation for VECM systems and shows that the matrix of (nonstructural) long-run coefficients, $\boldsymbol{C}(1)$, in equation (1) of the main paper is singular when derived from the VECM systems estimated by Beaudry and Portier (2006). This relationship holds not only in population, but also for any set of sample estimates of the underlying VECM coefficients. Moreover, $\boldsymbol{C}(1)$ has only rank 1 , implying that only one (independent) long-run restriction can be imposed on $\boldsymbol{C}(1)$. Since $\boldsymbol{\Gamma}_{0}$ is assumed to be nonsingular, the same properties hold for the sum of the structural VMA coefficients $\boldsymbol{\Gamma}(1)=\boldsymbol{C}(1) \boldsymbol{\Gamma}_{0}$.

Let $\boldsymbol{Y}_{\boldsymbol{t}}$ be a vector of nonstationary $I(1)$ variables, which are cointegrated such that $\boldsymbol{\alpha}^{\prime} \boldsymbol{Y}_{\boldsymbol{t}} \sim I(0)$ for some matrix of cointegrating vectors $\boldsymbol{\alpha}$. There is then a VECM representation:

$$
\Delta \boldsymbol{Y}_{\boldsymbol{t}}=\boldsymbol{F} \Delta \boldsymbol{Y}_{t-1}+\boldsymbol{G}\left(\boldsymbol{\alpha}^{\prime} \boldsymbol{Y}_{t-1}\right)+\boldsymbol{\mu}_{t}, \quad \boldsymbol{\mu}_{t} \sim i i d(\mathbf{0}, \boldsymbol{\Omega})
$$

For the sake of brevity, it is assumed that there is only a first-order lag dependence in the VECM, which can be easily generalized to higher-order cases. In addition, the notation abstracts from constants and other deterministic components of the data.

\footnotetext{
${ }^{1}$ Estimation results presented in Section 3 of our comment are derived from estimated VECM systems that include a constant and have been estimated with five lags, as in Beaudry and Portier (2006).
} 
The associated state-space representation is

$$
\boldsymbol{X}_{t}=\left[\begin{array}{r}
\Delta Y_{t} \\
\alpha^{\prime} Y_{t}
\end{array}\right]=\left[\begin{array}{cc}
F & G \\
\alpha^{\prime} \boldsymbol{F} & \boldsymbol{I}+\boldsymbol{\alpha}^{\prime} \boldsymbol{G}
\end{array}\right] \boldsymbol{X}_{t-1}+\left[\begin{array}{c}
\boldsymbol{I} \\
\boldsymbol{\alpha}^{\prime}
\end{array}\right] \boldsymbol{\mu}_{t}=\mathcal{A} \boldsymbol{X}_{t-1}+\mathcal{B} \mu_{t}
$$

And it follows the VMA representation:

$$
\Delta \boldsymbol{Y}_{\boldsymbol{t}}=\left[\begin{array}{ll}
\boldsymbol{I} & \mathbf{0}
\end{array}\right](\boldsymbol{I}-\mathcal{A} L)^{-1} \mathcal{B} \boldsymbol{\mu}_{t}=\boldsymbol{C}(L) \boldsymbol{\mu}_{t}
$$

\section{A.1 $C(1)$ has rank 1}

As will be shown later, the matrix of long-run coefficients $\boldsymbol{C}(1)$ is singular because of the assumed cointegrating relationships. In particular, we have $\boldsymbol{\alpha}^{\prime} \boldsymbol{C}(1)=0$, since $\boldsymbol{\alpha}^{\prime} \boldsymbol{C}(1)$ measures the long-run effect of a shock on the cointegrating vectors, which are stationary, and thus their long-run responses are zero (Hamilton, 1994). In the VECM systems used by Beaudry and Portier (2006), there are $N-1$ cointegrating relationships, and $\boldsymbol{\alpha}$ has $N-1$ columns when the VECM has $N$ variables. Thus, $\boldsymbol{C}(1)$ has only rank 1 .

The same holds also in sample for any point estimates of $\boldsymbol{F}, \boldsymbol{G}$, and $\boldsymbol{\alpha}$

- provided that $\mathcal{A}$ is stable. This result can be verified by computing the partitioned inverse of $\boldsymbol{I}-\mathcal{A}$ : 


$$
(\boldsymbol{I}-\mathcal{A})^{-1}=\left[\begin{array}{cc}
\boldsymbol{I}-\boldsymbol{F} & -\boldsymbol{G} \\
-\boldsymbol{\alpha}^{\prime} \boldsymbol{F} & -\boldsymbol{\alpha}^{\prime} \boldsymbol{G}
\end{array}\right]^{-1}=\left[\begin{array}{cc}
\boldsymbol{M}^{11} & \boldsymbol{M}^{12} \\
\boldsymbol{M}^{21} & \boldsymbol{M}^{22}
\end{array}\right]
$$

The standard formulas for the inverse of a partitioned matrix imply in this case that $\boldsymbol{M}^{12}=-\boldsymbol{M}^{11} G\left(\boldsymbol{\alpha}^{\prime} G\right)^{-1}$. Further, it follows that

$$
\boldsymbol{C}(1)=\boldsymbol{M}^{11}\left(\boldsymbol{I}-\boldsymbol{G}\left(\boldsymbol{\alpha}^{\prime} \boldsymbol{G}\right)^{-1} \boldsymbol{\alpha}^{\prime}\right) .
$$

And Sylvester's determinant theorem yields

$$
|\boldsymbol{C}(1)|=\left|\boldsymbol{M}^{11}\right|\left|\left(\boldsymbol{\alpha}^{\prime} \boldsymbol{G}\right)^{-1}\right|\left|\left(\boldsymbol{\alpha}^{\prime} \boldsymbol{G}-\boldsymbol{\alpha}^{\prime} \boldsymbol{G}\right)\right|=\mathbf{0} .
$$

Furthermore, it is straightforward to show that $\boldsymbol{\alpha}^{\prime} \boldsymbol{C}(1)=\mathbf{0}$ for any point estimates of $\boldsymbol{\alpha}, \boldsymbol{F}$, and $\boldsymbol{G}$. In order to verify this result, notice that

$$
\begin{aligned}
\boldsymbol{M}^{11} & =\left((\boldsymbol{I}-\boldsymbol{F})+\boldsymbol{G}\left(\boldsymbol{\alpha}^{\prime} \boldsymbol{G}\right)^{-1} \boldsymbol{\alpha}^{\prime} \boldsymbol{F}\right)^{-1} \\
& =(\boldsymbol{I}-\boldsymbol{F})^{-1}-(\boldsymbol{I}-\boldsymbol{F})^{-1} \boldsymbol{G}\left(\boldsymbol{\alpha}^{\prime}(\boldsymbol{I}-\boldsymbol{F})^{-1} \boldsymbol{G}\right)^{-1} \boldsymbol{\alpha}^{\prime} \boldsymbol{F}(\boldsymbol{I}-\boldsymbol{F})^{-1} \\
\Rightarrow \boldsymbol{\alpha}^{\prime} \boldsymbol{M}^{11} & =\boldsymbol{\alpha}^{\prime}(\boldsymbol{I}-\boldsymbol{F})^{-1}-\boldsymbol{\alpha}^{\prime} \boldsymbol{F}(\boldsymbol{I}-\boldsymbol{F})^{-1} \\
& =\boldsymbol{\alpha}^{\prime} \\
\Rightarrow \boldsymbol{\alpha}^{\prime} \boldsymbol{C}(1) & =\boldsymbol{\alpha}^{\prime} \boldsymbol{M}^{11}\left(\boldsymbol{I}-\boldsymbol{G}\left(\boldsymbol{\alpha}^{\prime} \boldsymbol{G}\right)^{-1} \boldsymbol{\alpha}^{\prime}\right) \\
& =\mathbf{0} .
\end{aligned}
$$


When $\boldsymbol{\alpha}$ has $N-1$ columns and $\boldsymbol{C}(1)$ is an $N \times N$ matrix, it follows that $\boldsymbol{C}(1)$ has rank 1.

\section{A.2 Long-run shocks to TFP in the VECM}

This section shows how to implement the identification of long-run shocks to TFP in the VECM systems. Throughout, a one-to-one mapping is assumed between forecast errors $\boldsymbol{\mu}_{t}$ and structural shocks $\varepsilon_{t}, \boldsymbol{\mu}_{t}=\boldsymbol{\Gamma}_{0} \varepsilon_{t}$, which must obviously satisfy $\boldsymbol{\Gamma}_{0} \boldsymbol{\Gamma}_{0}^{\prime}=\boldsymbol{\Omega}=E\left[\boldsymbol{\mu}_{t} \boldsymbol{\mu}_{t}^{\prime}\right]$.

For the VECMs considered by Beaudry and Portier (2006), there is a single common trend driving the permanent component of all variables, since there are $N-1$ cointegrating relationships when the system has $N$ variables. For the sake of convenience, the shock driving this trend will be referred to as long-run shocks to $T F P$, while it should be understood that the same shock also accounts for all long-run movements in $C, S P$, and potential other variables, denoted $X$. This section describes how to construct these long-run shocks from the reduced-form parameters of the VECM.

Consider the matrix of structural long-run responses $\boldsymbol{\Gamma}(1)=\boldsymbol{C}(1) \boldsymbol{\Gamma}_{0}$, and let the first column of $\boldsymbol{\Gamma}_{0}$ be the responses of forecast errors to the long-run shock. Since no other shock is issued to have a permanent effect on any of the VECM's variables, it follows that

$$
\Gamma(1)=\left[\begin{array}{ll}
\boldsymbol{x} & 0
\end{array}\right],
$$


where $\boldsymbol{x}$ denotes the column vector of long-run responses of $\boldsymbol{Y}_{\boldsymbol{t}}$ to the long-run shock.

A singular-value decomposition of $\boldsymbol{C}(1)$ yields

$$
\boldsymbol{C}(1)=\boldsymbol{V}\left[\begin{array}{cc}
\boldsymbol{S}_{1} & \mathbf{0} \\
\mathbf{0} & \mathbf{0}
\end{array}\right] \boldsymbol{W}^{\prime}=\boldsymbol{V}_{1} \boldsymbol{S}_{1} \boldsymbol{W}_{1}^{\prime}
$$

where $\boldsymbol{V}=\left[\begin{array}{ll}\boldsymbol{V}_{1} & \boldsymbol{V}_{2}\end{array}\right]$ and $\boldsymbol{W}=\left[\begin{array}{ll}\boldsymbol{W}_{1} & \boldsymbol{W}_{2}\end{array}\right]$ are conformably partitioned, unitary matrices, $\boldsymbol{V} \boldsymbol{V}^{\prime}=\boldsymbol{I}$ and $\boldsymbol{W} \boldsymbol{W}^{\prime}=\boldsymbol{I}$.

Without loss of generality, $\boldsymbol{\Gamma}_{0}$ can be written as the product of $\boldsymbol{W}$ and another matrix $\tilde{\boldsymbol{B}}$. As will be seen next, the long-run restriction requires that $\tilde{\boldsymbol{B}}$ is (block-) triangular:

$$
\tilde{\boldsymbol{B}}=\left[\begin{array}{cc}
\tilde{\boldsymbol{B}}_{11} & \tilde{\boldsymbol{B}}_{12} \\
\tilde{\boldsymbol{B}}_{21} & \tilde{\boldsymbol{B}}_{22}
\end{array}\right]=\left[\begin{array}{cc}
\tilde{\boldsymbol{B}}_{11} & 0 \\
\tilde{\boldsymbol{B}}_{21} & \tilde{\boldsymbol{B}}_{22}
\end{array}\right]
$$

The restriction $\tilde{\boldsymbol{B}}_{12}=0$ follows from $(\mathbb{6})$ and $(\mathbb{\nabla})$, since it ensures that

$$
W_{1}^{\prime} \Gamma_{0}=\left[\begin{array}{ll}
z & 0
\end{array}\right]
$$

where $z$ denotes an arbitrary column vector.

$\tilde{\boldsymbol{B}}$ factorizes $\tilde{\boldsymbol{\Omega}}=\boldsymbol{W}^{\prime} \boldsymbol{\Omega} \boldsymbol{W}$. A factorization of $\tilde{\boldsymbol{\Omega}}$ that satisfies the longrun restriction ([6) is the Cholesky factorization. The first column of $\boldsymbol{\Gamma}_{0}$ the column associated with the long-run shock — is then given by the first 
column of

$$
\Gamma_{0}=\boldsymbol{W} \operatorname{chol}(\tilde{\boldsymbol{\Omega}})
$$

and the long-run shocks are the first element of

$$
\varepsilon_{t}=\Gamma_{0}^{-1} \boldsymbol{\mu}_{t}
$$

where the remaining columns of $\boldsymbol{\Gamma}_{0}$, and thus also the remaining elements of $\varepsilon_{t}$, reflect an arbitrary permutation of the remaining shocks, without structural interpretation. For future use, the long-run shocks will be denoted $\bar{\varepsilon}_{t}$.

\section{B Multiple BP shock candidates}

The BP scheme for identifying news shocks hinges on two long-run restrictionsnamely, that one of the non-news shocks has zero effect on $T F P$ and $C$ in the long run. But as shown earlier, the matrix of long-run responses in the VECM's VMA representation is singular, with a rank of 1; one of these longrun restrictions is superfluous; and news shocks are not uniquely identified by the BP scheme. This section describes how to compute the set of candidate shocks in the VECM systems, which are consistent with the BP restrictions.

As an illustration, we re-estimate Beaudry and Portier's (2006) fourvariable VECMs with their original data and apply the procedure described 
here to obtain all possible impulse vectors that respect the $\mathrm{BP}$ restrictions and generate a positive impact response of the stock market. The results are reported in Section 2 of the comment, with further details presented in Section B.2 later.

\section{B.1 The entire set of solutions to the BP scheme}

To recap, the $\mathrm{BP}$ restrictions for the four-variable case are as follows:

1. There is a measurement error shock, which affects only the fourth variable in $\boldsymbol{Y}_{\boldsymbol{t}}$ on impact; depending on the VECM specification, this variable is either $H$ or $I$. The shock is denoted $\varepsilon_{t}^{4}$.

2. The "news shock," denoted $\varepsilon_{t}^{2}$ is orthogonal to TFP on impact.

3. There is a pure demand shock, denoted $\varepsilon_{t}^{3}$, which has no permanent effect on $T F P$ and $C$. (As argued earlier, this shock has, thus, no permanent effect on any of the VECM variables.)

In addition, all structural shocks are orthogonal to each other and have unit variance. Since the VECM has four variables, the three structural shocks

also imply a fourth "residual" structural shock, $\epsilon_{t}^{1}$, without any particular interpretation.

A candidate vector of structural shocks can simply be constructed by applying a series of projections using the forecast errors $\boldsymbol{\mu}_{t}$ and long-run shocks $\bar{\varepsilon}_{t}$ (see Appendix A.2) as follows: 
1. The variable $\varepsilon_{t}^{4}$ is the standardized residual in a regression of the fourth VECM residual, $\mu_{t}^{4}$, onto the other three residuals.

2. A "news shock" candidate can then be constructed as any linear combination of the VECM residuals, which is orthogonal to the forecast error for $T F P, \mu_{t}^{1}$, and the measurement error shocks $\varepsilon_{t}^{4}$. As will be shown later, it is then always possible to construct $\varepsilon_{t}^{3}$ with the desired properties. Because of the two orthogonality restrictions, only linear combinations in $\mu_{t}^{2}$ and $\mu_{t}^{3}$ need to be considered when constructing the news shock candidate. Specifically, we use a Givens rotation to construct $e_{t}=\sin (\theta) \mu_{t}^{2}+\cos (\theta) \mu_{t}^{3}$ and compute the news shock candidate as the standardized residual in regressing $e_{t}$ onto $\mu_{t}^{1}$ and $\varepsilon_{t}^{4}$. Different news shock candidates are thus indexed by the angle $\theta \in[0, \pi]$, denoted $\varepsilon_{t}^{2}(\theta)$. (Only the half-circle is considered, since the sign of the shock is determined by the restriction that it generates a positive stock market response on impact.)

3. For a given $\varepsilon_{t}^{2}(\theta)$, it is straightforward to compute a demand shock candidate, $\varepsilon_{t}^{3}(\theta)$, which has no permanent effect on the VECM variables. To ensure this long-run restriction, the demand shock must be orthogonal to $\bar{\varepsilon}_{t}$, as constructed in Appendix A.2, since $\bar{\varepsilon}_{t}$ is the sole driver of the permanent component in $\boldsymbol{Y}_{t}$. In addition, the demand shock has to be orthogonal to $\varepsilon_{t}^{4}$ and $\varepsilon_{t}^{2}(\theta)$. In sum, the demand shock candidate can be constructed as any linear combination of the VECM 
residuals that is orthogonal to $\varepsilon_{t}^{2}(\theta), \bar{\varepsilon}_{t}$, and $\varepsilon_{t}^{2}$. Since there are only four VECM residuals and there are three orthogonality constraints, any linear combination of the VECM residuals yields the same projection residual (up to scale and sign) — unless this linear combination should be perfectly spanned by $\varepsilon_{t}^{2}(\theta), \bar{\varepsilon}_{t}$, and $\varepsilon_{t}^{2}$, which is easy to check.

For a given candidate vector of shocks $\varepsilon_{t}(\theta)$, the corresponding candidate matrix $\boldsymbol{\Gamma}_{0}(\theta)$ is equal to the covariance matrix $E\left[\boldsymbol{\mu}_{t} \varepsilon_{t}(\theta)\right]$, which satisfies the $\mathrm{BP}$ restrictions by construction. All of these computations hold for both population and sample moments.

For the trivariate VECMs, the procedure is identical, except for the absence of $\varepsilon_{t}^{4}$. The set of BP candidate shocks is then described by any linear combination of the VECM residuals that is orthogonal to TFP on impact. Again, up to scale and sign, candidate shocks can be computed by projecting any linear combination of the residuals of $S P$ and $C$, denoted $\mu_{t}^{2}$ and $\mu_{t}^{3}$, off $\mu_{t}^{1}$

\section{B.2 Additional results from the BP VECMs}

Figure 1 of the main comment shows the range of impulse responses to all news shock candidates, which are consistent with the BP restrictions. Given the very different results across rotations, it should not come as a surprise that the range of correlation coefficients between the shocks satisfying the BP restrictions and the long-run $T F P$ shock is wide for both VECMs, ranging 
from about -0.50 to 0.99 . Likewise, as Table A. shows, the forecast error variance (FEV) shares of the different variables attributable to shocks consistent with the BP restrictions extend from basically $0 \%$ to above $80 \%$ for certain forecast horizons.

Each of these candidate solutions also implies different responses to the "demand shock," $\varepsilon_{t}^{3}$. As required, all of these solutions have zero effect on TFP and $C$ and-because of the assumed common trend in all variableson $S P$ and $H$. This fact is illustrated in Figure A.D, which depicts the set of impulse responses to the demand shock in each VECM at very long horizons. These results provide a computational consistency check that the $\mathrm{BP}$ restrictions indeed hold for the entire range of shock responses shown in Figure 1 of the main comment.

\section{References}

Paul Beaudry and Franck Portier. Stock prices, news, and economic fluctuations. The American Economic Review, 96(4):1293-1307, September 2006 .

James D. Hamilton. Time-Series Analysis. Princeton University Press, Princeton, NJ, 1994. 


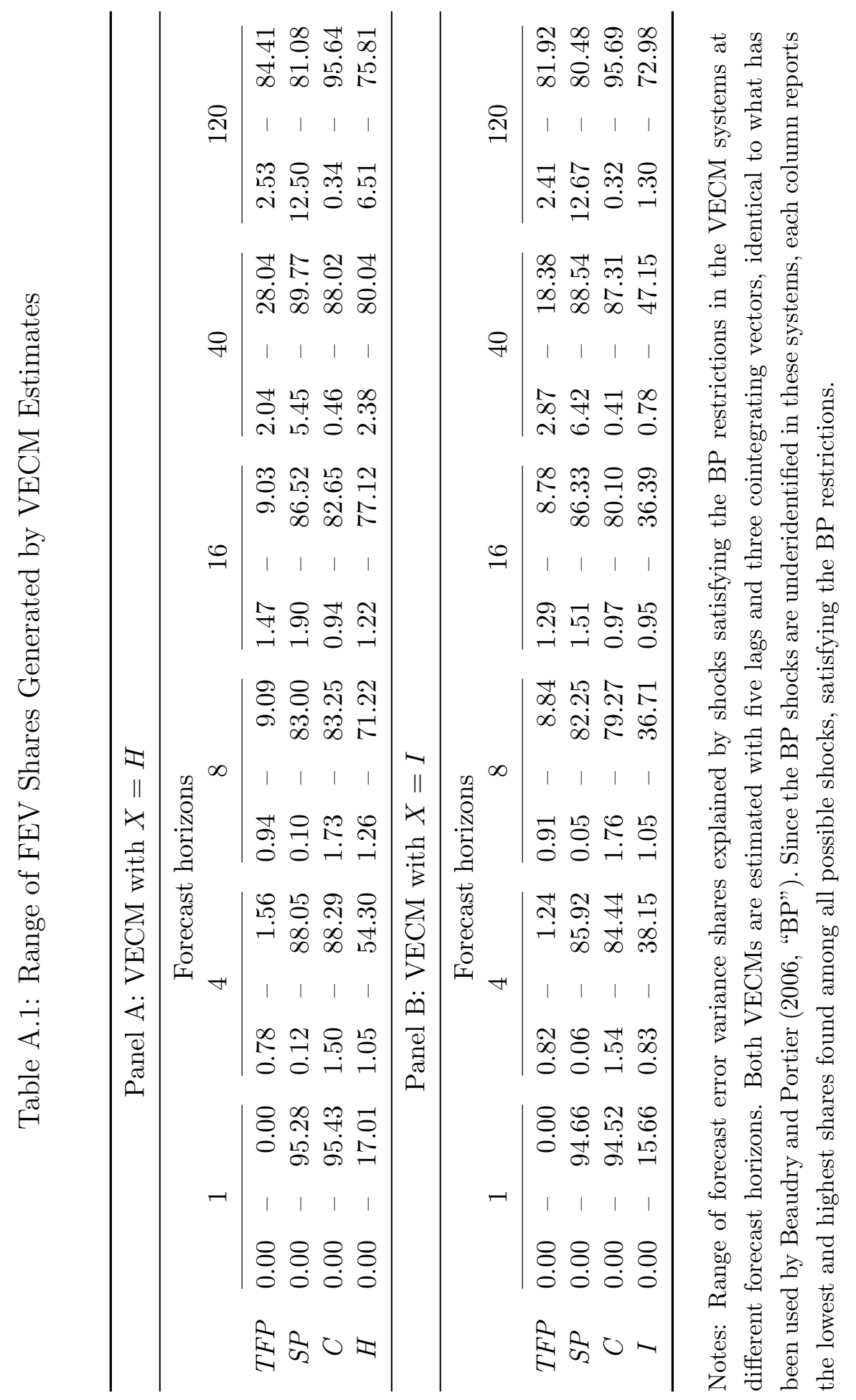




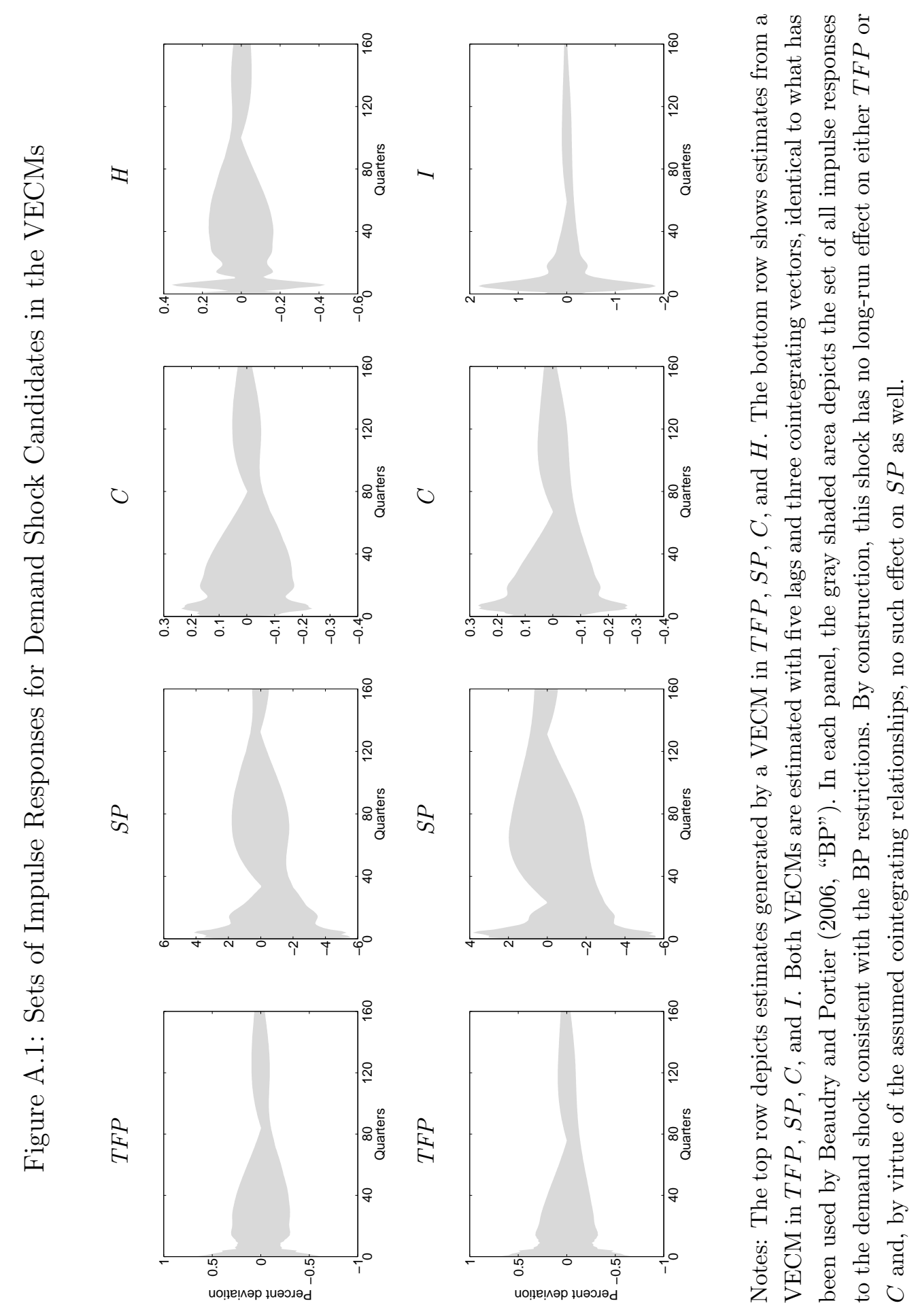

